\title{
NUCLEAR MODEL PREDICTIONS NEAR THE NEUTRON DRIP LINE RELEVANT TO R-PROCESS NUCLEOSYNTHESIS
}

\author{
Y. JADING ${ }^{1}$, J. DOBACZEWSKI ${ }^{2}$, V.N. FEDOSEYEV ${ }^{3}$, K.-L. KRATZ ${ }^{1}$, \\ V.I. MISHIN ${ }^{3}$, P. MÖLLER ${ }^{4}$, B. PFEIFFER ${ }^{1}$, H.L. RAVN ${ }^{5}$, F. SCHEERER ${ }^{1}$, \\ F.-K. THIELEMANN ${ }^{6}$, W.B. WALTERS ${ }^{7}$, A. WÖHR ${ }^{1}$ AND THE ISOLDE \\ COLLABORATION ${ }^{3}$ \\ ${ }^{1}$ Institut für Kernchemie, Universität Mainz, D-55099 Mainz, Germany \\ ${ }^{2}$ Institute of Theoretical Physics, Warsaw University, 00-681 Warsaw, Poland \\ ${ }^{3}$ Institute of Spectroscopy, Russian Academy of Sciences, 142092 Troitzk, Russia \\ 4 Theoretical Division, Los Alamos National Laboratory, Los Alamos, \\ NM 87545, USA \\ ${ }^{5}$ CERN, CH-1211 Geneva 23, Switzerland, \\ ${ }^{6}$ Institut für Theoretische Physik, Universität Basel, CH-4056 Basel, Switzerland \\ ${ }^{7}$ Department of Chemistry and Biochemistry, University of Maryland, \\ $M L$ 20742, USA

\begin{abstract}
Using the waiting-point approximation, which assumes the classical $(\mathrm{n}, \gamma) \rightleftharpoons(\gamma, \mathrm{n})$-equilibrium, in combination with a local $\beta$-flow equilibrium, we have been able to reproduce the solar r-process abundances $\left(N_{r, \odot}\right)$ fairly well with a minimum of three neutron-density components. However, local deficiencies have been observed around the abundance peaks. They have been interpreted as being due to weaknesses in the description of the shell-structure near the neutron drip line. In this contribution, it will be shown that the deficiencies can be considerably reduced when using new data from experiments at CERN/ISOLDE together with recent mean-field calculations of nuclear masses according to the HFB-method with the Skyrme P interaction.
\end{abstract}

\section{Introduction}

Neutron capture is predicted to be the dominant process of nucleosynthesis for elements beyond Fe. This is strongly supported by the fact that the elemental abundances for our solar system contain three twin peaks correlated with the magic neutron numbers $\mathrm{N}=50,82$ and 126 . The observed double structures indicate that the nucleosynthesis due to neutron capture can be divided into two processes taking place under different stellar conditions: (i) The slow neutron-capture process (s-process) occurs during He-burning in Red Giants with moderate neutron densities $\left(\mathrm{n}_{n} \simeq 10^{8} \mathrm{~cm}^{-3}\right)$ and goes through long-lived isotopes close to stability. (ii) The rapid neutron-capture process ( $r$-process) is suggested to occur in type II supernovae (SN II) with high neutron densities $\left(\mathrm{n}_{n} \geq 10^{20} \mathrm{~cm}^{-3}\right)$, synthesizing progenitor isotopes well off the vailey of $\beta$-stability. Despite its importance, the r-process is still poorly understood. This is closely connected with the fact that the very neutron-rich isotopes - formed during this process are out of reach for experiments in terrestrial laboratories. 
Only recently, first experimental information in the $A \simeq 80$ and 130 regions ${ }^{1-4}$ made it possible to analyze isotopic abundance patterns in these two $\mathrm{r}$-peaks ${ }^{5,6}$ within the waiting-point concept ${ }^{7,8}$.

'Complete' r-process network calculations require a large number of astrophysical and nuclear-physics input parameters. In order to facilitate these complicated calculations, during the past three decades many attemps to predict the $N_{\tau, \odot}$ distribution were based on the simplified assumptions of the $(n, \gamma) \rightleftharpoons(\gamma, n)$ equilibrium concept $^{7-15}$. When assuming in addition a steady-flow equilibrium of $\beta$-decays, the prediction of $r$-abundances requires only the input of nuclear masses (respectively neutron separation energies, $\left.S_{n}\right), \beta$-decay half-lives $\left(T_{1 / 2}\right)$ and $\beta$-delayed neutron emission probabilities $\left(P_{n}\right)$, as well as the stellar parameters temperature $\left(T_{9}\right)$, neutron density $\left(n_{n}\right)$ and process duration $(\tau)$. Whereas for a given $\mathrm{n}_{n}$, the $S_{n}$ determine the r-process path, the $T_{1 / 2}$ of the isotopes along this flow path, in principle, define the progenitor abundances and - when taking into account $P_{n}$ branching - also the final $r$-abundances. Only in recent years, the validity of this waiting-point approximation in combination with a steady $\beta$-decay flow could be confirmed locally for the $A \simeq 80$ and $130 N_{r, \odot}$ peaks on the basis of first experimental information in the r-process path ${ }^{6}$. With this, the long $T_{1 / 2}$ of the classical $N=82$ waiting-point nucleus ${ }^{130} \mathrm{Cd}$ is, for example, directly correlated with the large $N_{r, \odot}$ value of its isobar ${ }^{130} \mathrm{Te}$ in the $A \simeq 130 \mathrm{r}$-abundance peak. The astrophysical exact site of the r-process still remains unclear, but lately it has been agreed that the r-process is likely to take place in a so-called high-entropy bubble in type II supernovae ${ }^{16-20}$.

\section{Nuclear Data for R-Process Calculations}

Since the vast majority of nuclei in or close to the $\mathrm{r}$-process path will probably never become accessible in terrestrial laboratories, a general understanding of their nuclear properties can only be obtained through theoretical means. In order to avoid a vanishing of real signatures or the creation of artificial $r$-abundance effects from the use of mass and half-life models of largely different sophistication ${ }^{17,18,21}$, we have tried to perform the calculations in a unified approach ${ }^{22-24}$ within which all relevant nuclear properties can be studied in an internally consistent way. The combination of nuclear masses from the Finite-Range Droplet Model (FRDM) ${ }^{25}$ and $\beta$-decay properties from the QRPA approach of Moller and Randrup ${ }^{26}$ is discussed in detail in ${ }^{22,23}$. Analogously, when adopting the masses from the Extended Thomas-Fermi plus Strutinski Integral (ETFSI) model ${ }^{27}$, we use $\beta$-decay properties deduced from QRPA calculations with the $Q_{\beta}$ values and deformation parameters given by this approach. For comparison with the above two macroscopic-microscopic theories, in special cases we also use the macroscopic formula of Hilf et al. ${ }^{28}$, which is the only droplet-type model with a steep mass parabola.

In principle. with the above two global approaches - FRDM+QRPA and 
ETFSI +QRPA - we have two rather sophisticated, and internally consistent nucleardata sets for astrophysical calculations which are expected to yield more reliable predictions of nuclear-physics parameters than earlier models. Nevertheless, being aware that even these approaches must have their deficiencies, we have tried to improve the data sets by taking into account all recent experiments on $Q_{\beta}, S_{n}, T_{1 / 2}$ and $P_{n}$. Furthermore, for localized extrapolations known nuclear-structure properties, either model-inherently not contained in or not properly described by the above global methods, were taken into account.

\section{Fits to Solar Abundances}

We have shown ${ }^{22,23}$ that a superposition of three time-dependent components, where each had attained a steady-flow equilibrium between magic numbers, and which fitted each a $N_{r, \odot}$ peak and the adjacent interpeak region for lower mass numbers, gave already a quite impressive fit to the solar r-process abundances. The conditions were taken from a 'best fit' with static steady-flow assumptions to each of the above three mass regions. In fact, we had combined the global FRDM masses ${ }^{25}$ with the masses of Hilf et al. ${ }^{28}$ for the $Z=39$ to 53 region $^{22}$, in order to avoid to some degree the otherwise huge abundance trough at $A \simeq 115$ before the $N=82$ shell closure.

We do observe a slight improvement from the three-component to the tencomponent superposition, but the major deviations persist: a trough around $A \simeq 115$ 125 before the $A \simeq 130$ peak and two spikes at $A \simeq 112$ and $A \simeq 176$. The first deviation is essentially explained by a mass-formula effect (i.e., a too strong shell-effect when approaching $N=82$ ); and the two spikes are caused by deformation-dependent too long $\beta$-decay half-lives in transitional regions. Hence, the above result is a clear proof that our three-component fit ${ }^{22}$ is a quite valid first-order approach, and that for reasonable superpositions which follow a natural continuum of stellar conditions, a quite good but not perfect fit is obtainable. The remaining deviations can, in fact, be utilized to set constraints on necessary nuclear-model features far from stability. This will be discussed in more detail later.

\subsection{Dependence on Nuclear Masses}

In Fig. 1, we show global r-abundance curves from a superposition of ten $n_{n}-\tau$ components for three different nuclear mass sets with the corresponding QRPA calculations for $T_{1 / 2}$ and $P_{n}$ values. In all three cases, identical conditions for the stellar parameters were used.

As already discussed above, the FRDM+Hilf 'hybrid' masses used in the upper part of Fig. 1 yield the best possible agreement with the $N_{r, \odot}$ distribution. In the middle part of Fig. 1, exclusively FRDM mass predictions are used. Apart from a 

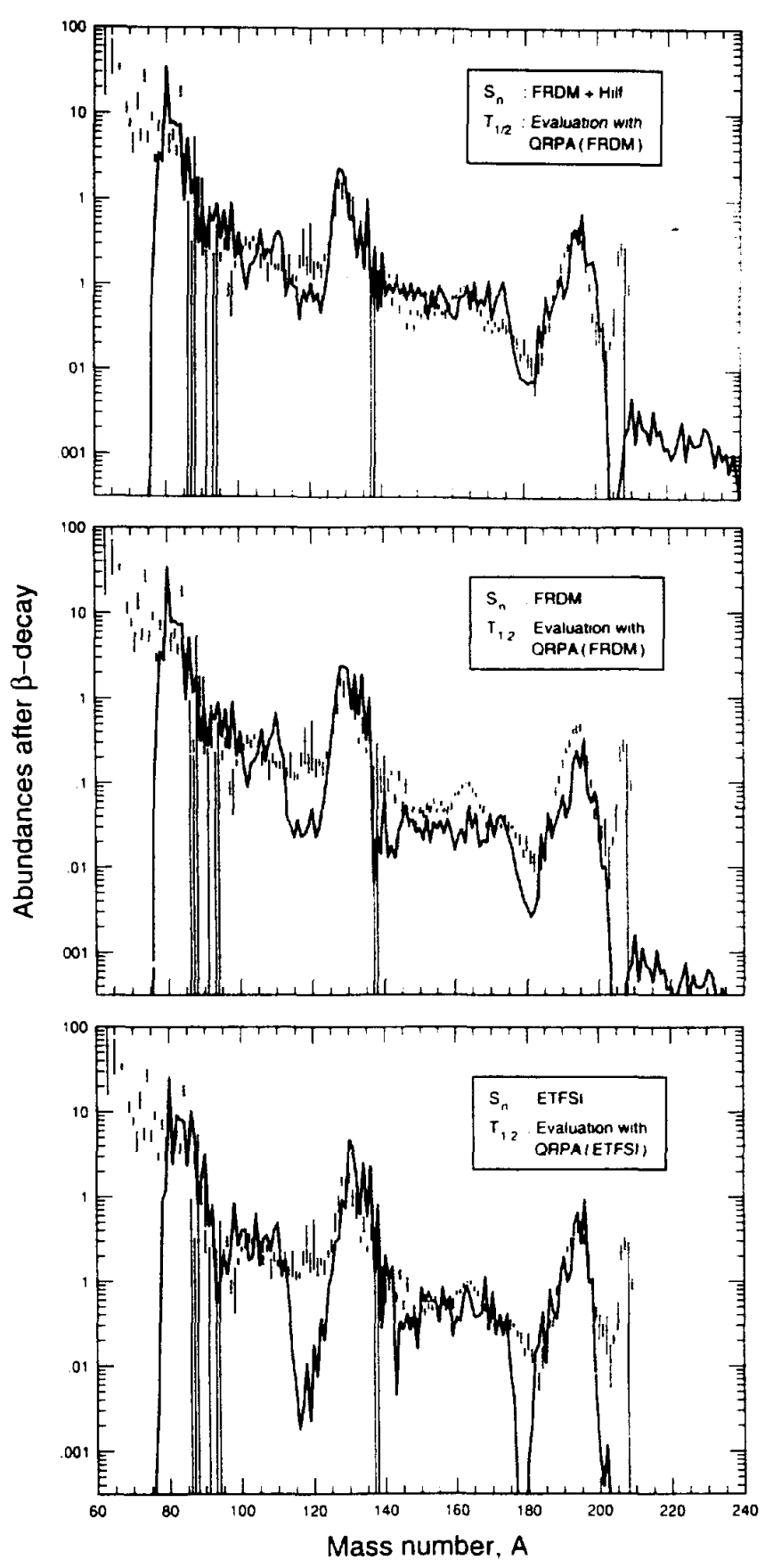

Figure 1: Global r-abundance fits with superpositions of ten r-components, calculated with the same $n_{n}$ $T_{9}-\tau$ conditions. Upper part: $S_{n}$ values and input for the QRPA calculations from FRDM ${ }^{25}$ below $Z=39$ and beyond $Z=53$, and in the intermediate region from the Hilf et al. ${ }^{28}$ mass formula. Middle part: $S_{\mathrm{n}}$ values and input for the QRPA calculations from FRDM ${ }^{25}$ : lower part: $S_{n}$-values and input for QRPA from ETFSI ${ }^{27}$. For discussion, see text.

more pronounced $A \simeq 115$ abundance trough, under the given $n_{n}-\tau$ conditions too little r-material is observed beyond the $A \simeq 130$ peak. This is due to a number of obviously too low $S_{n}$ values in the r-process path just beyond the $N=82$ shell, which act as a bottle-neck in the r-process flow. In the lower part of Fig. 1, the results from the ETFSI masses plus 3-decay properties are shown. Here, pronounced abundance troughs at $A \simeq 115$ and 175 occur due to overly strong $N=82$ and $N=126$ shell 
corrections. As has already been discussed ${ }^{22,23}$, a consequence of the pronounced shell strengths is a wrong trend in the $S_{n}$ values beyond neutron mid-shells. This behaviour of the FRDM and ETFSI masses implies that in certain localized regions there would exist not a single isotope in the r-process path. The resulting abundance troughs cannot be avoided, neither by using an even more continuous distribution of superpositions, nor by choosing $\mathrm{r}$-components closer to $\beta$-stability with neutron densities down to $n_{n} \simeq 10^{17} \mathrm{~cm}^{-3}$. The same deviations are also observed in the recent astrophysically realistic r-process calculations of neutrino-heated SN II ejecta ${ }^{16.18-20}$, which is a strong indication that they are not due to stellar-model deficiencies but signatures of new nuclear-structure effects very far from $\beta$-stability.

\section{Improvement of Nuclear-Structure Description far from Stability}

Nuclear shell-structure between $\beta$-stability and unstable regions half-way to the r-process path are reasonably well studied by now, both experimentally and theoretically. Partly due to the recent developments in radioactive ion-beam (RIB) physics, the yet unknown structure of nuclei near and at the particle drip-lines has become one of the most exciting challenges today. Although the parameters of interactions used so far in mean-field theories were mainly determined as to reproduce known properties, these models have a surprisingly high global predictive power for unknown exotic nuclei (for nuclear-masses and $\beta$-decay properties see e.g. ${ }^{29}$ and ${ }^{26}$, respectively). However, for the sometimes dramatic extrapolations to the limits of particle binding, the above parameters may not always be proper to be used at the drip-lines, in particular near classical shell closures. Therefore, new spectroscopic results from ISOL or RIB experiments far off stability will be essential to test the model predictions. Having in mind, however, that such exotic isotopes are only accessible in exceptional cases, our approach of learning drip-line structure from astrophysical observables, as are the r-process abundances, will become even more important for a better understanding of nuclear forces and interactions depending on isospin degrees of freedom.

\subsection{New Information on Beta-Decay Properties}

The shell model, with its single-particle (s-p) basis and residual interactions, is fundamental to nuclear structure. Largely due to the proton-neutron $(\mathrm{p}-\mathrm{n})$ interaction among the valence nucleons, the s-p level energies change across the periodic table. The effect of the monopole part of the p-n interaction has been discussed by Heyde

${ }_{30}$ at the example of the neutron s-p levels of ${ }_{40}^{91} \mathrm{Zr}_{r 1}$ and ${ }_{50}^{131} \mathrm{Sn}_{81}$. With the filling of the $\pi g_{9 / 2}$ shell a dramatic lowering of the $\nu g_{7 / 2}$ orbit (by up to $3 \mathrm{MeV}$ ) occurs, leading even to an inversion of the $\nu d_{5 / 2}$ and $\nu g_{7 / 2}$ states in the ${ }^{132} \mathrm{Sn}$ region. Consequences 
Table 1: Comparison of experimental half-lives $\left(T_{1 / 2}\right)$ of neutron-rich Ag isotopes with literature values and QRPA predictions ${ }^{31}$.

\begin{tabular}{|c||c|c|c|c|}
\hline \multirow{2}{*}{ A } & \multicolumn{4}{c|}{ Beta-decay half-life, $T_{1 / 2}[\mathrm{~ms}]$} \\
\cline { 2 - 5 } & \multicolumn{2}{|c|}{ Experiment } & \multicolumn{2}{c|}{ Theory } \\
\cline { 2 - 5 } & This work & Literature & QRPA-Nilsson & QRPA-F.Y. \\
\hline \hline 121 & $1045(40)$ & $910(60)$ & 216 & 405 \\
122 & $528(11)$ & $480(80)$ & 102 & 261 \\
123 & $297(6)$ & $309(15)$ & 60 & 117 \\
124 & $171(10)$ & $590(80)$ & 51 & 100 \\
125 & $156(7)$ & - & 49 & 117 \\
126 & $97(8)$ & - & 62 & 153 \\
127 & $109(15)$ & - & 36 & 80 \\
\hline
\end{tabular}

of this effect on the $\beta$-decay properties (in particular on the $T_{1 / 2}$ ), and on the validity of the waiting-point concept for the $A \simeq 130 \mathrm{r}$-abundance peak have been discussed in 22.23 .

In the context of studying the influence of the $\mathrm{p}-\mathrm{n}$ interaction on $T_{1 / 2}$ values, an experiment to identify neutron-rich $\mathrm{Ag}$ isotopes has recently been carried out at CERN/ISOLDE ${ }^{31}$. The isotopes were produced by proton-induced fission of ${ }^{238} \mathrm{U}$ in a $\mathrm{UC}_{2}$-target. In order to avoid disturbing isobars, a chemically selective laser ion source (LIS) was applied. The Ag atoms diffused into a niobium capillary where they were excited in two resonant steps with dye-lasers and non-resonantly ionized with a high power copper vapor laser in a third step, and were then mass separated. The detector set-up consisted of a $4 \pi{ }^{3} \mathrm{He}$-neutron counter and a scintillator for $\beta$-detection. Although the astrophysically important waiting-point nucleus ${ }^{129} \mathrm{Ag}_{82}$ could not yet be observed unambiguously, the previously known $T_{1 / 2}$ of ${ }^{121-124} \mathrm{Ag}$ were improved and the $T_{1 / 2}$ of ${ }^{125-127} \mathrm{Ag}$ were measured for the first time. For all these isotopes, longer $T_{1 / 2}$ were obtained than predicted by our early straightforward QRPA calculations neglecting the $\mathrm{p}-\mathrm{n}$ interaction. In Table 1 , the measured half-lives are compared with literature values and with two sets of QRPA shell-model predictions. The first data set represents results from old calculations using Nilsson-model wave functions and neglecting effects from the $\mathrm{p}-\mathrm{n}$ residual interaction. In the second set, the more recent Folded-Yukawa s-p model with an isospin-dependent energy term is used which takes into account the above $\mathrm{p}-\mathrm{n}$ interaction effect in an empirical way. With the observed $T_{1 / 2}$ trend, a rather reliable prediction of $T_{1 / 2} \simeq(120-140) \mathrm{ms}$ is now possible for the $N=82$ waiting-point isotope ${ }^{129} \mathrm{Ag}$, in good agreement with our old astrophysical request of roughly $160 \mathrm{~ms}^{6}$ deduced from the $A \simeq 130 N_{r, \odot}$ peak shape. As an example of possible effects on $r$-abundance fits due to improved nuclear-physics input, Fig. 2 shows the results of a static calculation with $N_{r, \odot}(Z) \lambda_{\beta}(Z)=$ const. for the $A \simeq 130$ peak. In the left part, the fit obtained when using the straightforward $T_{1 / 2}$ predictions from our old QRPA is displayed. The right part shows the 
calculated r-abundances derived from recently measured $T_{1 / 2}$ and $P_{n}$ values of $\mathrm{Ag}$, $\mathrm{Cd}$ and In isotopes, together with improved QRPA predictions using Folded-Yukawa or experimental s-p levels. It is clearly evident from this figure, that a considerable improvement of the $A \simeq 130 \mathrm{r}$-abundance fit can be achieved with the updated nuclearphysics input. As has already been discussed qualitatively in ${ }^{22}$, other effects due to the neglection of the $\mathrm{p}-\mathrm{n}$ residual interaction in both the mass models and the QRPA formalism to calculate GT strength functions (from which the theoretical $T_{1 / 2}$ and $P_{n}$ values are derived) are the deficiencies in the development of quadrupole deformation before and beyond neutron mid-shells ( $N=66$ and 104). A first, more quantitative

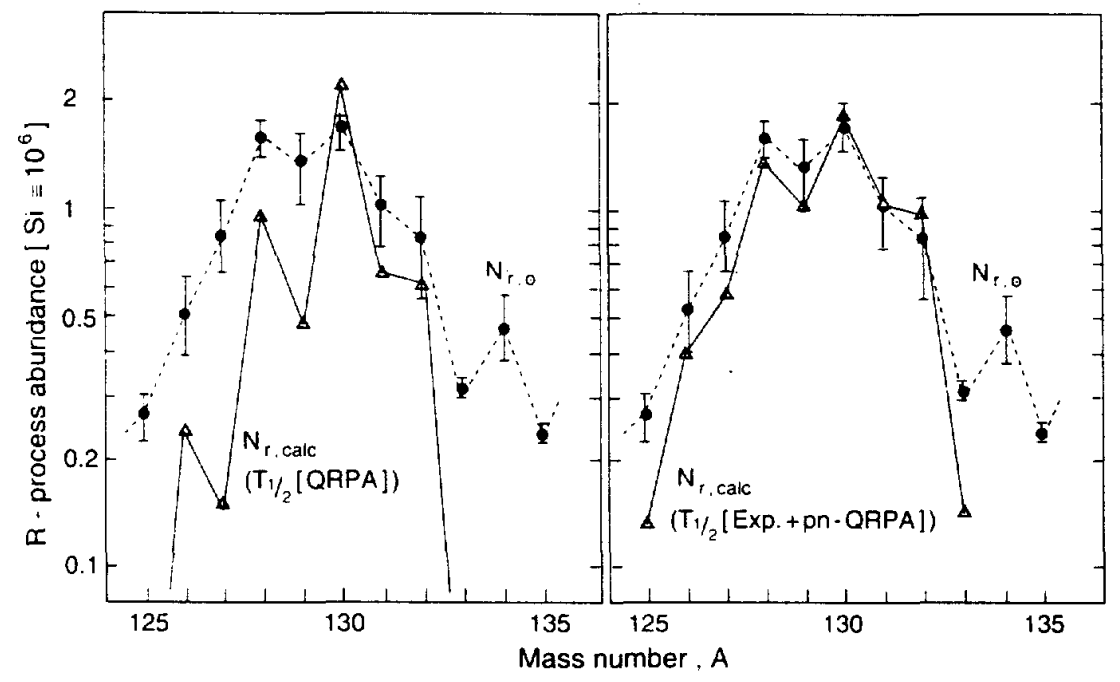

Figure 2: Static steady-flow fits $\left[N_{r, \odot}(Z) \lambda_{\beta}(Z)=\right.$ const.] for the $A \simeq 130$ r-abundance peak. In the left part, $T_{1 / 2}$ and $P_{n}$ values from the old straightforward QRPA calculations. neglecting effects from the $\mathrm{p}-\mathrm{n}$ interaction, are used. The right part shows a considerably improved fit, resulting from the application of measured $\beta$-decay properties together with new QRPA predictions with an empirical s-p energy correction. For further discussion, see ${ }^{22,23}$.

interpretation for the $A \simeq 120$ mass region in terms of a weakening of the $\pi f_{5 / 2}-\nu h_{11 / 2}$ residual interaction. simultaneously affecting $S_{n}$ and $T_{1 / 2}$ values, was given by Walters 32. More recently, similar consequences for drip-line nuclei have been discussed in terms of neutron-skin effects within state-of-the-art mean-field theories ${ }^{33,34}$. With the $\mathrm{p}-\mathrm{n}$ residual interaction being stronger than the $\mathrm{p}-\mathrm{p}$ and $\mathrm{n}-\mathrm{n}$ interactions, the proton potential becomes deeper when going away from $\beta$-stability, whereas the potential for neutrons becomes shallower and more diffused. As in the picture of Walters ${ }^{32}$, this leads to the formation of a loosely-bound $\nu h_{11 / 2}$ neutron skin in the $A \simeq 120$ mass region, accompanied by a vanishing of the shell gap at $N=82$. It has already been discussed in ${ }^{22}$. that another consequence for the exotic $40 \leq Z \leq 44, N \geq 72$ nuclides will be a less deformed shape as the skin neutrons feel smaller restoring forces against 
quadrupole deformation than the inner core neutrons. We can also show, that this leads to longer half-lives as well as changes in the $P_{n}$ values.

It is worth to be mentioned in this context, that in all other mass regions where the r-process path does not come close to the neutron drip-line - the $T_{1 / 2}$ will not be affected and, therefore, will not modify the present $r$-abundance fits.

\subsection{New Information on Neutron Separation Energies}

As is discussed in some detail in ${ }^{22.23}$, a reproduction of the $N_{r, \odot}$ pattern in the vicinity of neutron shell closures requires a quenching of shell-effects close to the neutron drip-line which is obviously not contained in the global mass models ${ }^{25,27}$ used so far. That such a request is not purely speculative, has already been shown for the $N=28$ and 50 shells in ${ }^{35}$ and ${ }^{36}$, respectively. Since meanwhile, the shape coexistence around ${ }_{16}^{44} \mathrm{~S}_{28}$ has been confirmed by Skyrme-HF calculations ${ }^{37}$, it seems worthwhile to check the results from such state-of-the-art mean-field models also for the $N=50,82$ and 126 shells.

In a self-consistent, microscopic way nuclei can be described by the densitydependent Skyrme mean-field or by the relativistic mean-field (RMF) theory. In two recent papers ${ }^{33,38}$, the behaviour of shell-effects in nuclei near the neutron drip-line have been examined within these approaches, leading to considerably different results. Whereas in the RMF ${ }^{38}$, strong shell-effects for $N=82$ are obtained supporting the predictions of the FRDM and ETFSI mass models, the Skyrme $\mathrm{P}$ ansatz ${ }^{33}$ results in a quenching of shell-effects as requested from our astrophysical considerations ${ }^{22,23}$. The contradictory results obviously originate from a different treatment of pairing correlations in drip-line nuclei. With the neutron Fermi energy being close to the top of the neutron potential well, the pairing force scatters part of the quasiparticles into continuum states. As the centrifugal barrier pushes up states of high angular momentum $\mathrm{j}$. the energies of low-j states decrease relative to all other levels. In light and medium-heavy nuclei, low-j continuum states, which are located right above the shell gaps, enter these gaps and effectively lead to quenched shell-effects. For the $N=82$ region, this is shown in Fig. 3 at the example of the $S_{n}$ values below ${ }_{50}^{132} \mathrm{Sn}_{82}$. From the energy distance $\left[S_{n}(N=81)-S_{n}(N=83)\right]$, it is evident that the FRDM and the ETFSI which use BCS or BCS-like pairing methods, show a very strong $N=82$ shell effect. In these approaches, ${ }_{40}^{122} \mathrm{Zr}_{82}$ would be (nearly) as neutronmagic as ${ }_{50}^{132} \mathrm{Sn}_{82}$. Only the Skyrme $\mathrm{P}$ ansatz with the new treatment of the pairing force results in a quenching of shell-effects.

With the promising features of the latter mean-field model ${ }^{33}$, we have started to perform static steady-flow r-abundance fits for the 'pathological' $A \simeq 115$ and 175

mass regions. Since so far the Skyrme $\mathrm{P}$ variant is limited to the calculation of the 


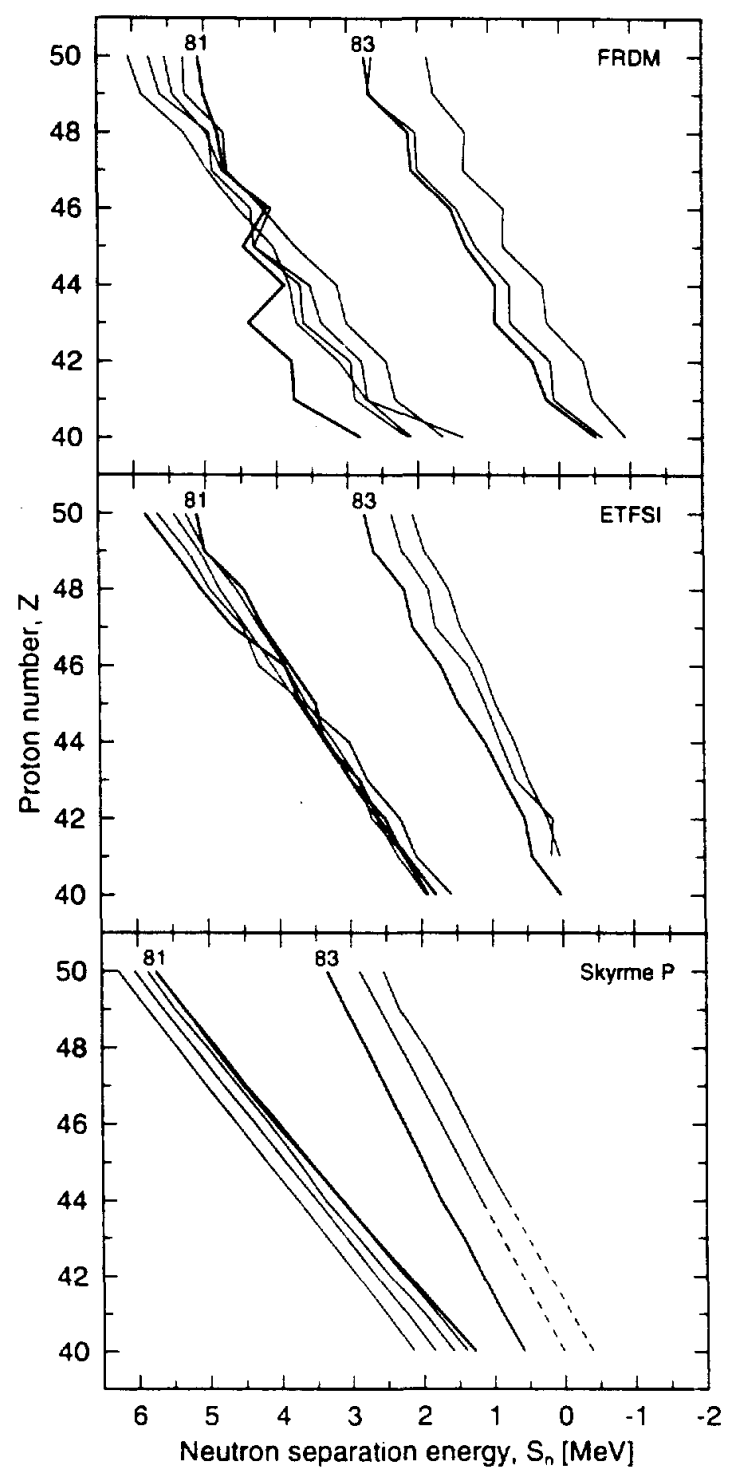

Figure 3: Neutron separation energies $\left(S_{n}\right)$ for odd-N isotones as obtained from different mass models, upper part: FRDM ${ }^{25}$, middle part: ETFSI ${ }^{27}$ and lower part: Skyrme $\mathrm{P}{ }^{33}$. The energy distance $\left[S_{n}(N=81)-S_{n}(N=83)\right]$ between the isotones is a measure for the $N=82$ shell strength. Only the Skyrme P ansatz with the new treatment of the pairing force leads to the required quenching of the $N=82$ shell-effect.

spherical shape, we only have replaced the mass predictions from the global FRDM ${ }^{25}$ and ETFSI ${ }^{27}$ models locally by the new $S_{n}$ 's around the magic $N=82$ and 126 shells. Under exactly the same $n_{n}-T_{9}$ conditions as requested for our 'best fits' in ${ }^{22}$, we indeed observe a filling up of the abundance troughs to a large extent. A preliminary result for the $90 \leq A \leq 130$ mass region (the second component with $n_{n}=9.5 \times 10^{20} \mathrm{~cm}^{-3}$ and $\left.T_{9}=1.20\right)$ is shown Fig. 6 in ${ }^{24}$.

As has been discussed in ${ }^{22.23}$, due to the too slow decrease of the $S_{n}$ 's of neutron-rich $Z>40$ isotopes in the FRDM and ETFSI models, not a single nuclide with $S_{n} \simeq 2 \mathrm{MeV}$ would exist in the r-process path just below $N=82$. With the steeper $S_{n}$ trend of the Skyrme P model, however, altogether seven isotopes (with $Z=40$ to 


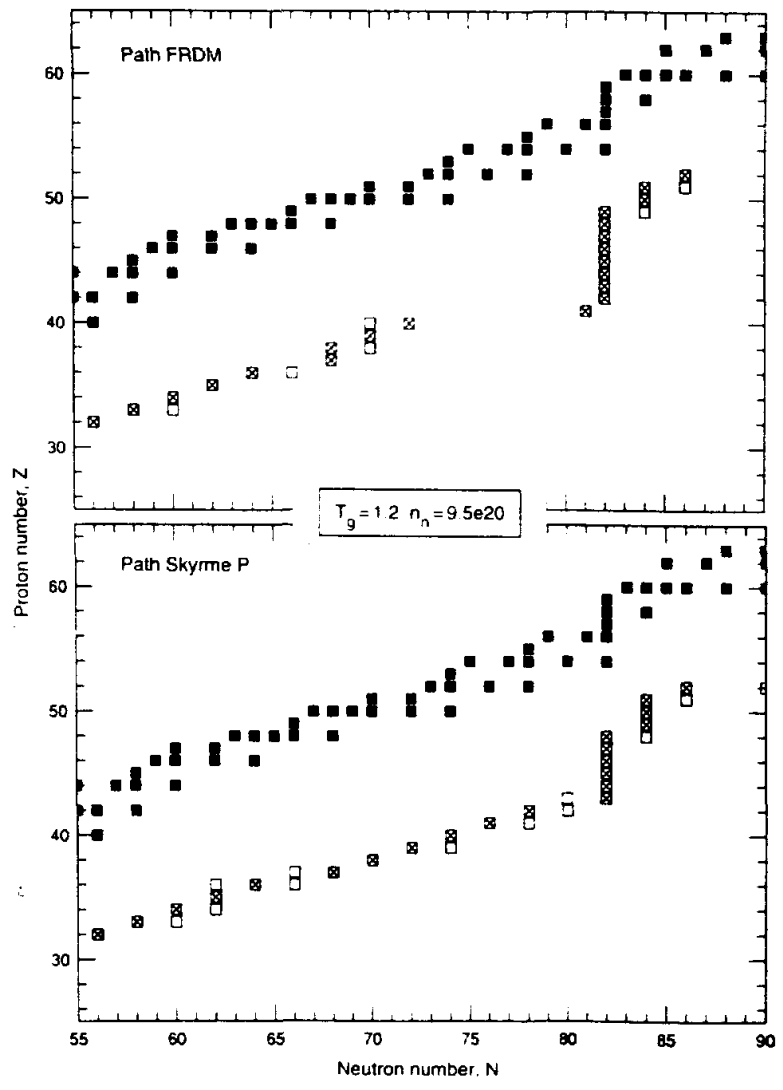

Figure 4: Isotopes in the r-process path, top part: $S_{n}$ values from FRDM ${ }^{25}$, bottom part: $S_{n}$ values from Skyrme $\mathrm{P}^{33}$.

43 , and $N=74$ to 80 ) become potential progenitors, see Fig. 4. Their initial abundances are now sufficient to nearly reproduce the $N_{r, \odot}$ pattern in the $A \simeq 115-125$ mass region. If, in addition to the new $\mathrm{S}_{n}$ values, also the longer $T_{1 / 2}$ discussed above will be used, a very good fit may be expected. A detailed analysis of multicomponent, time-dependent $r$-abundance calculations with the improved nuclear-physics input is in progress ${ }^{39}$.

\section{Summary}

New experimental results together with recent mean-field calculations indicate partly new nuclear-structure signatures of neutron drip-line nuclei which seem to be preserved in the $N_{r, \odot}$ pattern. Our deductive approach has given valuable constraints on stellar parameters, excluding explosive He-burning and supporting the hot-entropy scenario of SN II explosions. On the other hand, it has helped to initiate recent improvements of nuclear-structure models. A deeper understanding of the coupling between nuclear physics at the drip-lines and explosive nucleosyntheses will certainly remain an exciting challenge for future work. 


\section{Acknowledgements}

The authors would like to thank M. Krieg, T. Mehren and T. Rauscher for their assistance in the silver experiment at CERN/ISOLDE, K. Langanke and W. Nazarewicz for discussions, and J.M Pearson for making available the ETFSI masses. Financial support from BMFT (06 MZ 465), and DFG ( $\mathrm{Kr} 806 / 3$ and $436 \mathrm{RUS} 17 / 26 / 93$ ) is gratefully acknowledged.

\section{References}

1. K.-L. Kratz et al., Z. Phys. A325 (1986) 483.

2: B. Ekström et al., Phys. Scr. 34 (1986) 614.

3. R.L. Gill et al., Phys. Rev. Lett. 56 (1986) 1874.

4. K.-L. Kratz et al., Z. Phys. A340 (1991) 419.

5. K.-L. Kratz et al., J. Phys. G14 (1988) 331.

6. K.-L. Kratz, Rev. Mod. Astron. 1 (1988) 184.

7. E.M. Burbidge et al., Rev. Mod. Phys. 29 (1957) 547.

8. A.G.W. Cameron, Atomic Energy of Canada, Ltd. CRL-41 (1957).

9. P.A. Seeger et al., Ap. J. Suppl. 97 (1965) 121.

10. A.G.W. Cameron et al., CERN-Report 70-30 (1970) 735.

11. T. Kodama and K. Takahashi, Nucl. Phys. A239 (1975) 489.

12. W. Hillebrandt, Space Sci. Rev. 21 (1978) 639.

13. J.W. Truran et al., Ap. J. Lett. 222 (1978) L63.

14. F.-K. Thielemann et al., Astron. Astrophys. 74 (1979) 175.

15. A.G.W. Cameron, Astrophys. Space Sci. 91 (1983) 235.

16. B.S. Mever et al., Ap. J. 399 (1992) 656.

17. W.M. Howard et al., Ap. J. 417 (1993) 713.

18. K. Takahashi et al., Astron. Astrophys. 286 (1994) 857.

19. S.E. Woosley and R. Hoffman, Ap. J. 395 (1992) 202.

20. S.E. Woosley et al., Ap. J. (1994), in print.

21. S. Goriely and V. Bouquelle, in Nuclei in the Cosmos, ed. F. Käppeler and K. Wisshak (IOP, London. 1992).

22. K.-L. Kratz et al., Ap. J. 403 (1993) 216. 
23. K.-L. Kratz et al., in Nuclei in the Cosmos, ed. F. Käppeler and K. Wisshak (IOP, London, 1992).

24. K.-L. Kratz. in Nuclei in the Cosmos (AIP, New York, 1994) in print.

25. P. Möller et al., At. Data Nucl. Data Tables (1994) in print.

26. P. Möller, and J. Randrup, Nucl. Phys. A514 (1990) 1.

27. Y. Aboussir et al., Nucl. Phys. A549 (1992) 155.

28. E.R. Hilf et al., in NFFS-3. CERN 76-13 (1976) 142.

29. P. Möller and J.R. Nix, in NFFS-6 and AMCO-9 (IOP, London, 1993).

30. K. Heyde, in Nuclear Structure of the Zirconium Region, ed. J. Eberth et al. (Springer, Berlin, 1988).

31. V.N. Fedoseyev et al.. in Resonance Ionisation Spectroscopy and its Applications, ed. G. Huber et al. (AIP, New York, 1994) in print.

32. W.B. Walters, in Nuclear Physics of our Times (WS, Singapore. 1993).

33. J. Dobaczewski et al., Phys. Rev. Lett. 72 (1994) 981; and priv. com.

34. N. Fukunishi et al., Phys. Rev. C48 (1993) 1648.

35. O. Sorlin et al., Phys. Rev. C47 (1993) 2941.

36. K.-L Kratz et al., Phys. Rev. C38 (1988) 278.

37. T.R. Werner et al., Phys. Lett. B333 (1994) 303.

38. M.M. Sharma et al., Phys. Rev. Lett. 72 (1994) 1431.

39. B. Chen et al., submitted to Phys. Lett. B (1994). 